\title{
Mobility reduction in the flow of an elastic microcapsule through a constricted channel
}

\author{
Jose F. Roca, Ivan F. Menezes, and Marcio S. Carvalho* \\ Department of Mechanical Engineering, Pontifícia Universidade Católica do Rio de \\ Janeiro, RJ 22453-900, Brazil \\ E-mail: msc@puc-rio.br
}

\section{Supporting Information}

The line integral of eq.(19) is evaluated using a 1-D mesh that approximates the membrane configuration at each time step:

$$
\int_{0}^{l s(t)} T \mathbf{t} \cdot h d \mathbf{W}_{i}(\mathbf{X})=\sum_{e=1}^{N e} h T(e) \mathbf{t}(e) \cdot\left[\mathbf{W}_{i}\left(\mathbf{X}_{e+1}\right)-\mathbf{W}_{i}\left(\mathbf{X}_{e}\right)\right]
$$

where $e$ is a 1-D membrane element and $N e$ is the number of elements in the 1-D domain. It is important to note that $\mathbf{W}_{i}\left(\mathbf{X}_{k}\right), k=e, e+1$ is non-null only if the solid domain node $k$ is inside a 2-D element $f_{e}$ at which the weighting function $\mathbf{W}_{i}$ is non-null. The line integral is only evaluated along the elements at which the weighting function $\mathbf{W}_{i}$ is non-null, as sketched in Fig.S1. Evaluating $\mathbf{W}_{i}\left(\mathbf{X}_{k}\right)$ requires mapping a point defined in global coordinates to elemental coordinates. We used a quadratic inverse mapping for this purpose, which requires finding $(\xi, \eta)$ for a given $(x, y)$, as sketched in Fig. (S2).

To do so, a second-order Taylor polynomial of $(x, y)$ about the point $\left(x_{9}, y_{9}\right)$, center of the Eulerian element, is used: 


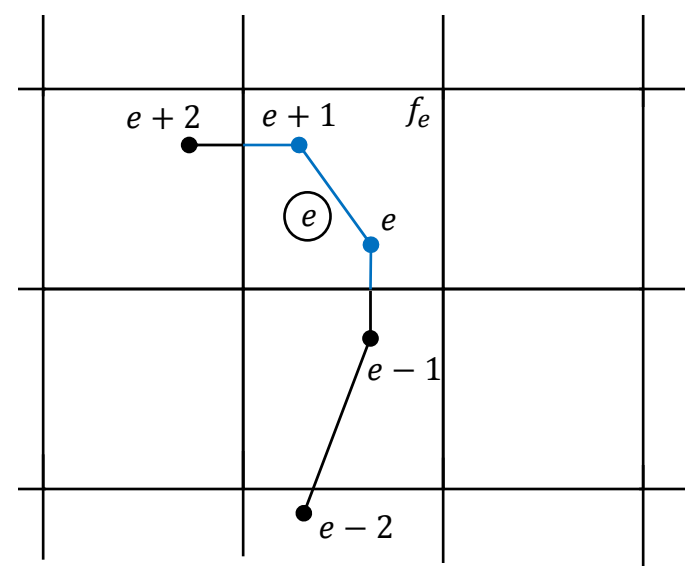

Figure S1: Discretization of structure domain (line segments) and fluid domain (quadrilaterals).

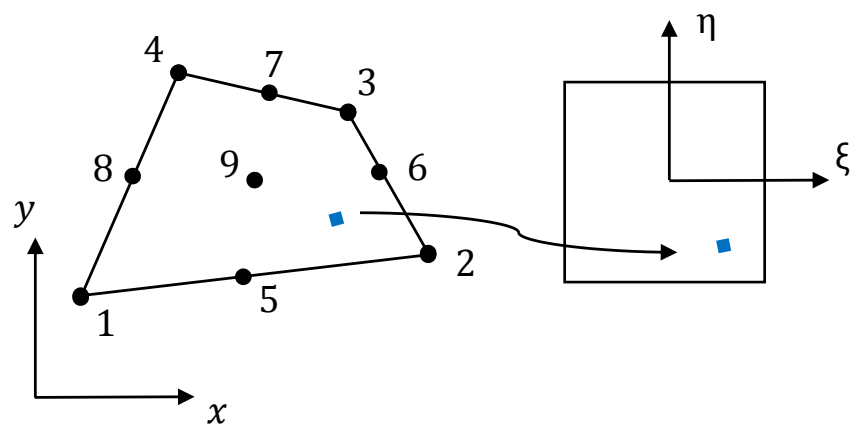

Figure S2: Given a global position, find the local coordinates.

$$
\begin{aligned}
x-x_{9}= & \left.\frac{\partial x}{\partial \xi}\right|_{0} \Delta \xi+\left.\frac{\partial x}{\partial \eta}\right|_{0} \Delta \eta+\frac{1}{2}\left[\left.\frac{\partial^{2} x}{\partial \xi^{2}}\right|_{0} \Delta \xi^{2}\right. \\
& \left.+\left.\frac{\partial^{2} x}{\partial \eta^{2}}\right|_{0} \Delta \eta^{2}+\left.2 \frac{\partial^{2} x}{\partial \eta \partial \xi}\right|_{0} \Delta \eta \Delta \xi\right] \\
y-y_{9}= & \left.\frac{\partial y}{\partial \xi}\right|_{0} \Delta \xi+\left.\frac{\partial y}{\partial \eta}\right|_{0} \Delta \eta+\frac{1}{2}\left[\left.\frac{\partial^{2} y}{\partial \xi^{2}}\right|_{0} \Delta \xi^{2}\right. \\
& \left.+\left.\frac{\partial^{2} y}{\partial \eta^{2}}\right|_{0} \Delta \eta^{2}+\left.2 \frac{\partial^{2} y}{\partial \eta \partial \xi}\right|_{0} \Delta \eta \Delta \xi\right]
\end{aligned}
$$

where $\Delta \xi=\xi-0$ and $\Delta \eta=\eta-0$.

Since $x=x(\xi, \eta), y=y(\xi, \eta)$ and their respective first and second derivatives are known, 
a multivariable nonlinear system is obtained to find $(\xi, \eta)$ for a given $(x, y)$. This system is properly solved using Newton's method.

As time elapses and the capsule deforms, it is possible that a 1-D solid element $e$ crosses a 2-D fluid element $f_{e}$ without having a solid node located inside the fluid element, as illustrated in Fig.S3. In this case, an extra solid node is included in the 1-D mesh inside element $f_{e}$ to compute the line integral using eq.(1). The extra node is located in the middle of segment $(a, b)$, that defines the portion of $e$ inside element $f_{e}$.

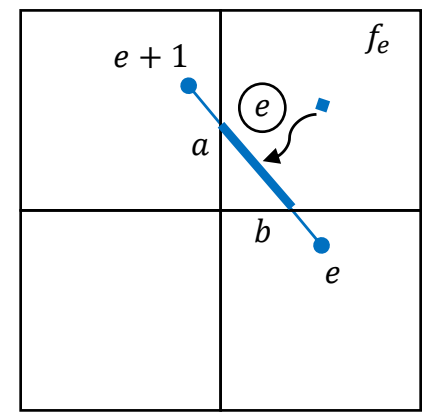

Figure S3: Sketch of situation at which an extra node is included to compute the line integral along the membrane domain. 